\title{
IR investigations of surfaces and adsorbates
}

\author{
Gwyn P Williams \\ Thomas Jefferson National Accelerator Facility, 12000 Jefferson Avenue, Newport News, \\ VA 23606, USA
}

Received 26 June 2001

Published 23 November 2001

Online at stacks.iop.org/JPhysCM/13/11367

\begin{abstract}
Synchrotron infrared reflection-absorption measurements on single crystal metal surfaces with adsorbates have led to the determination of many key parameters related to the bonding vibrational modes and the dynamics of adsorbates. In particular, energy couplings between electrons and adsorbate motion have been shown to be a dominant mechanism on metal surfaces. Excellent agreement has been obtained with calculations for many of the observations, and the synergy between theory and experiment has led to a deeper understanding of the roles of electrons and phonons in determining the properties of interfaces and their roles in phenomena as diverse as friction, lubrication, catalysis and adhesion. Nonetheless, as the experiments are pushed harder, to describe such effects as co-adsorbed systems, disagreements continue to challenge the theory and our comprehension also is still evolving.
\end{abstract}

\section{Introduction}

Infrared light is a potentially powerful probe of surfaces, since it covers a wide range of energies at high energy resolution, while studying the system essentially in the ground state, albeit it is as close to zero momentum transfer. The development of the synchrotron as an infrared source has enabled experiments to be performed at much lower frequencies, while the absolute nature of this new source has revealed new and unexpected phenomena, which have themselves led to a more fundamental understanding of surface dynamics. These include the breakdown of the so-called dipole selection rule, which used to be a rigid rule describing how dynamic dipoles perpendicular to a metal surface were the only ones that could be excited by either infrared light or a grazing incidence beam (p-polarized light). A breakdown of Born-Oppenheimer rules, which assert that electrons follow nuclear motion, is implicit in the above statement.

The motivation for developing infrared synchrotron radiation was the study of adsorbate bonding and intermolecular dynamics. Prior to its development, much of the information about the nature of the bonds at surfaces, and even the relaxation mechanisms, was being inferred from measurements of the intramolecular vibrational modes in the $600-4000 \mathrm{~cm}^{-1}$ frequency range [1-6]. Using synchrotron radiation one can measure at lower frequencies 
and study not only these intramolecular modes but also the bonding modes in the 20-600 $\mathrm{cm}^{-1}$ range. These are crucial in any attempt to understand the many macroscopic phenomena involving interfacial processes such as adhesion, adsorption, desorption, diffusion, friction, lubrication, epitaxial growth, catalysis and electronic properties.

Infrared studies of adsorbates on metal surfaces require a very bright infrared source to compensate for the lack of energy available at the sample from standard thermal sources and in particular to overcome the $300 \mathrm{~K}$ thermal background that limits detector performance. In some cases these thermal problems have been overcome [7] by cooling all the optical components, but this does not work if the sample itself is warm enough to contribute to detector thermal background. Infrared synchrotron radiation is about 1000 times brighter than globar sources [8], and therefore offers considerably improved signal-to-noise capabilities. It is also an absolute source, since the power depends on the electron beam current stored in the ring which can be measured accurately. This latter advantage has opened up a whole new field of surface dynamics, studying effects that had hitherto not even been noticed in IR spectroscopy such as the broadband reflectivity loss measured for various adsorbates on $\mathrm{Cu}$, which had passed un-noticed for almost two decades, despite many measurements in the frequency range over which it is found to occur. Synchrotron light is also highly polarized, and pulsed on the 100 ps timescale, properties which have not been utilized in these experiments, but certainly will be in the future.

Although synchrotron radiation has many advantages, the exploitation of this long wavelength radiation requires some special engineering considerations since large angles are required to extract it. Also, special attention has to be paid to the motion and size fluctuations of the electron beam, since very small changes in chemical properties are usually being measured. Due to the intrinsic dynamic dipole moment of the molecules and their low concentration (especially at the sub-monolayer level) spectral reproducibilities of 1 part in $10^{4}$ in a minute measuring time are usually required, which is much more stringent than the 1 part in $10^{2}$ which, for example, is typically used in photoemission experiments. Difficulties of achieving these high levels of reproducibility were problematic at first and delayed the early exploitation of this source. However, with many of these problems solved in 1990, infrared synchrotron radiation has enjoyed over a decade of research applications, although we still strive for further improvements in source stability.

We begin this chapter with a discussion of some of the essential details which are necessary in the utilization of infrared synchrotron radiation.

\section{Experimental details}

\subsection{Signal-to-noise and absolute IR spectroscopy}

In most spectroscopies, but especially for the infrared spectral region, one is concerned with signal-to-noise and we have paid considerable attention to this issue. For this spectral region one can use Michelson interferometers to perform Fourier transform infrared (FTIR) experiments. For such an instrument, where the limit is detector noise, the signal-to-noise ratio $S / N$ per scan can be written as [9]:

$$
S / N=\frac{B(\bar{\nu})(\delta \bar{\nu}) \Theta \xi D^{*}}{(\Delta f)^{1 / 2} A^{1 / 2}}
$$

where $B(\bar{v})$ is the source brightness, $\delta \bar{v}$ the resolution, $\Theta$ the limiting etendue of the experiment, $\xi$ the optical efficiency, $D^{*}$ the detectivity, $\Delta f$ the electronic bandwidth and $A$ the area of the detector. Here it is assumed that the etendue, $\Theta$, the (area $\times$ solid angle) product describing the sample, is less than that of the source. Clearly, there is an upper 


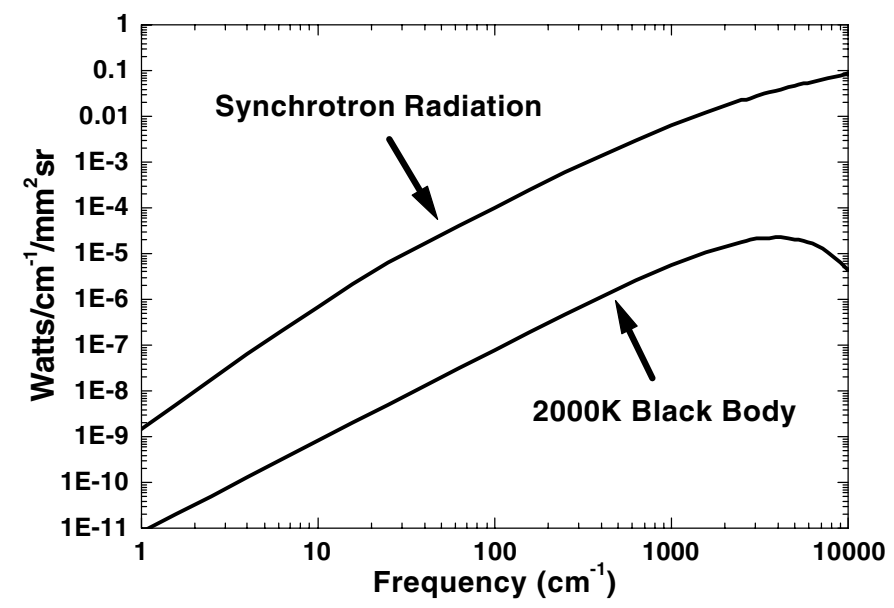

Figure 1. Calculated brightness values for synchrotron radiation, specifically the U4IR beamline at the NSLS, Brookhaven, with $800 \mathrm{~mA}$ of beam, and a $2000 \mathrm{~K}$ thermal source assuming an emissivity of unity.

bound on $\Theta$ and for surface science it is quite small. Therefore, even with the best detectors, superior signal-to-noise is possible only if the source brightness can be increased. Brightness, sometimes called spectral brilliance, is defined as the power into a given spectral bandwidth, per unit area-angle product, and thus is in units of $\mathrm{W} \mathrm{mm} \mathrm{mm}^{-2} \mathrm{sr}^{-1}$. In figure 1 we show the advantage of synchrotron radiation in a plot which compares the brightness of synchrotron radiation with that of a standard thermal source.

Since the synchrotron source brightness is 1000 times greater than that for thermal sources, equation (1) tells us that, all other things being equal, to attain similar quality spectra with the latter, the data collection time would need to be a million times longer, i.e. a $1 \mathrm{~s}$ measurement would take 11 days! We have discussed signal-to-noise issues in detail before [10] and while calculations lead one to expect to be able to obtain $S / N$ ratios of $10^{5}$ with the synchrotron, compared to 10-100 with the globar, in practice we have found that this is not fully achieved due to instabilities in the electron orbits in the synchrotron. These instabilities are of several kindslong term drifts probably due to changes in temperature as the storage ring current decays, short term fluctuations in the $10-100 \mathrm{~Hz}$ region due to mechanical vibrations and power supplies, and radio-frequency (rf) side-bands in the kilohertz region from the accelerating cavity. Standard rapid-scan FTIR spectrometers and detectors are sensitive to noise in the audio range of frequencies, so reducing the beam instabilities is crucial for realizing the brightness advantage of the source. We addressed the low $(<300 \mathrm{~Hz})$ instabilities by installing a global feedback system [11] on the NSLS VUV ring in which the electron orbit is measured and corrected using special additional magnets. The rf side-bands were reduced [12] through careful tuning of the synchrotron's rf cavity, minimizing their effects on the spectral noise. Continued improvements in reducing these noise sources will benefit all the infrared programs.

Apart from the reproducibilities (noise) of $0.01 \%$ in 1 min of measuring time at the NSLS, absolute reflectance changes can be measured. This is because the brightness of synchrotron radiation is dependent only on the magnitude of the stored beam current which can be measured to a part in $10^{6}$. Variables normally present in IR spectroscopy with a thermal source, such as temperature fluctuations and emissivity changes, do not exist. In practice, the absolute accuracy of the present experiments was found to be $\pm 0.2 \%$ across a broad spectral band from $300 \mathrm{~cm}^{-1}$ to $3000 \mathrm{~cm}^{-1}$ using a silicon beamsplitter and a liquid helium cooled $\mathrm{Cu}$ doped $\mathrm{Ge}$ 
photoconductive detector, provided the data were all taken within $10 \mathrm{~min}$ or so. Over longer periods of time, as the current decayed in the storage ring, there were slight orbit shifts due to changing thermal loads on the synchrotron, which led to higher discrepancies. However, it will be possible to eliminate these changes using continual injection into the storage ring, the so-called top-off mode, in which the stored beam current is maintained close to about $500 \mathrm{~mA}$ by frequent injections. We have tested this and managed to get spectra reproducible on an absolute scale to $\pm 0.2 \%$ for periods of over an hour.

\subsection{Synchrotron radiation sources}

In a previous paper [13, and references therein], we have discussed the instrumentation indepth. The most important experimental details are of an engineering nature and involve dealing with the large opening angles of the synchrotron radiation at long wavelengths. Practical issues make it difficult to extract the infrared, particularly from storage rings of large radius. The 'natural opening angle', while less than a milliradian at the critical wavelength, increases to several tens of milliradians in the infrared depending on the radius of the ring as follows [8]:

$$
\theta_{\text {nat }}(\operatorname{rad})=1.66\left(\frac{\lambda}{\rho}\right)^{1 / 3}
$$

where $\lambda$ is the wavelength and $\rho$ the radius (in the same units as $\lambda$ ). $\theta_{\text {nat }}$ is the angle required to transmit $90 \%$ of the emitted light at a given wavelength. Note that although this formula shows that the radiation opening angles are smaller for larger rings, engineering, thermal problems at the first optic and radiation shielding difficulties make it more practical to utilize smaller rings. Also the source depth is larger for rings of larger radius making it more difficult optically. At the NSLS, $\rho=1.91 \mathrm{~m}$, and opening angles of $90 \times 90 \mathrm{mrad}$ were used for the far infrared beamlines.

Infrared beamlines differ from VUV lines in that ultrahigh vacuum is not required except for beam extraction. Pressures of 100 mTorr are sufficient to eliminate problems due to water vapour and carbon dioxide. Ideally, the infrared beamlines are separated from the storage ring vacuum chambers by wedged diamond windows which are usually placed near a mirror focus. The emerging beams are collimated or re-focused into a Michelson interferometer. The present work was carried out at resolutions down to $1 \mathrm{~cm}^{-1}(125 \mathrm{meV})$. Wedged diamond windows were not available for the work described here. Instead, the white synchrotron beam was focused either through a $250 \mu \mathrm{m}$ diamond or a $6 \mathrm{~mm}$ thick CsI window, then collimated into a Nicolet Impact 400 rapid scan Michelson interferometer equipped with a silicon beamsplitter and modified for use in vacuum. Mirror scanning velocities were a few $\mathrm{mm} \mathrm{s}^{-1}$ or equivalently, the HeNe reference laser was modulated at frequencies of up to $50 \mathrm{kHz}$. The latest NSLS beamline utilizes a vacuum modified Nicolet Nexus FTIR modulator with a resolution down to $0.025 \mathrm{~cm}^{-1}$ and with modulation frequencies of up to $125 \mathrm{kHz}$.

\subsection{Synchrotron radiation and surface science}

The experimental situation at the NSLS, Brookhaven, is typical of the surface science experiments using synchrotron radiation. The beam emerging from the Michelson interferometer was refocussed $(f / 10)$ into an ultra high vacuum (UHV) chamber with a base pressure of $1 \times 10^{-10}$ Torr, and reflected at a grazing incidence angle of $\sim 87^{\circ}$ off a sample of typical dimensions $25 \mathrm{~mm} \times 5 \mathrm{~mm}$, the throughput being $6.5 \times 10^{-2} \mathrm{~mm}^{2} \mathrm{sr}^{-1}$. The ultrahigh vacuum was separated from the 100 mTorr beamline vacuum by either $6 \mathrm{~mm}$ CsI or by polyethylene windows. A boron-doped silicon bolometer at $4.2 \mathrm{~K}$ was used to detect light 
in the $50-600 \mathrm{~cm}^{-1}$ range, while a $\mathrm{Cu}$-doped $\mathrm{Ge}$ photoconductor was used for frequencies of $350 \mathrm{~cm}^{-1}$ and above. This arrangement gave reproducibilities of $1 \times 10^{-4}$ at a resolution of $1 \mathrm{~cm}^{-1}$ over the $50-2500 \mathrm{~cm}^{-1}$ range in $3 \mathrm{~min}$ of measuring time. The CsI windows allowed spectra to be taken to a resolution of $1 \mathrm{~cm}^{-1}$ in the range above $180 \mathrm{~cm}^{-1}$. For frequencies below this, un-wedged diamond was used as the beamline window and this restricted the resolution to $6 \mathrm{~cm}^{-1}$ due to interference fringes.

For the work which was done using $\mathrm{Cu}$ single crystals at the NSLS, the samples were first prepared with several cycles of mechanical and electro-polishing which is described in detail elsewhere [14]. Subsequent electron channelling patterns, acquired from a secondary electron microscope (JSM 6400) operated at $25 \mathrm{keV}$ and $6^{\circ}$ grazing incidence, revealed well-defined Kikuchi bands, indicating good crystal quality in the surface region. Once in vacuum, the crystals were cleaned by sputtering at $300 \mathrm{~K}$ with a $0.08^{\circ} \mu \mathrm{A} \mathrm{mm}^{-2}$ beam of $500 \mathrm{eV} \mathrm{Ne}^{+}$ions for $20 \mathrm{~min}$. They were rotated to grazing incidence $\left(60^{\circ}\right.$, both directions) not only to increase the sputtering efficiency but also to cover the $25 \mathrm{~mm}$ long surface more uniformly. The crystals were then briefly annealed at $850 \mathrm{~K}$. Auger spectra showed no sulfur, carbon or oxygen peaks, with an upper limit on the detection of $<2 \%$ of the surface-sensitive copper $(105 \mathrm{eV})$ peak, which corresponds to less than $1 \%$ of a monolayer of contamination. If small amounts of carbon were detected, the intensity of the low frequency modes was noticeably reduced. These electropolished samples produced better low energy electron diffraction (LEED) patterns after the first sputter and anneal cycle in the UHV chamber and much narrower and stronger adsorbate peaks in the IR than a mechanically polished surface that had been cleaned in UHV for six months.

The thin $\mathrm{Cu}\left(\begin{array}{lll}1 & 1 & 1\end{array}\right)$ films were grown epitaxially on $\mathrm{TiO}_{2}\left(\begin{array}{lll}1 & 1 & 0\end{array}\right)$ crystals [15], and were prepared in a separate chamber as follows. First, the polished $\mathrm{TiO}_{2}$ crystals were annealed by resistive heating for $5 \mathrm{~h}$ at $973 \mathrm{~K}$. After cooling down to room temperature, thicknesses between 20 and $100 \mathrm{~nm}$ of copper were evaporated at a rate of $0.1 \mathrm{~nm} \mathrm{~s}^{-1}$. These asgrown films exhibited LEED patterns with slightly diffuse spots. After preparation of the $15 \mathrm{~mm} \times 10 \mathrm{~mm}$ films, four $200 \mathrm{~nm}$ thick copper pads were evaporated onto the edges to allow measurement of the films' resistance $W$. The absolute resistance values were determined with a four-terminal method. In order to register small changes $\Delta W$ of film resistance, the films were made part of a Wheatstone bridge which was supplied with alternating current. A lock-in amplifier was used to improve the signal-to-noise ratio.

The film resistance at room temperature was also used to determine the thickness of the clean films by comparison to a calibration curve. This was not trivial because at first there is no film to measure. Thus, in the initial growth process, the film thickness was determined with a quartz microbalance. Then the samples were removed, contacts were put on, and the samples re-mounted in UHV and cleaned by ion bombardment. During the subsequent epitaxial regrowth process (see below), the resistance and the additional film thickness, again using the quartz microbalance, were recorded continuously. If $W_{\text {start }}$ and $W_{\text {end }}$ are the start and end values of the sheet resistance (at $T=293 \mathrm{~K}$ ) in this process and if the known thickness change is $\Delta d$, the film thickness $d$, corresponding to the resistance $W_{\text {start }}$, can be determined by

$$
d=\frac{\Delta d}{W_{\text {start }} / W_{\text {end }}-1}
$$

neglecting diffuse scattering at the interface itself.

Thus two points $d\left(W_{\text {end }}\right)$ and $d\left(W_{\text {start }}\right)$ for the calibration curve were obtained. The samples were then withdrawn from the UHV chamber in Düsseldorf, stored in glass vessels containing dry nitrogen, and shipped to the NSLS for mounting in the U4IR chamber on a manipulator, which allows cooling to liquid nitrogen temperature (around $90 \mathrm{~K}$ ). One hour of 

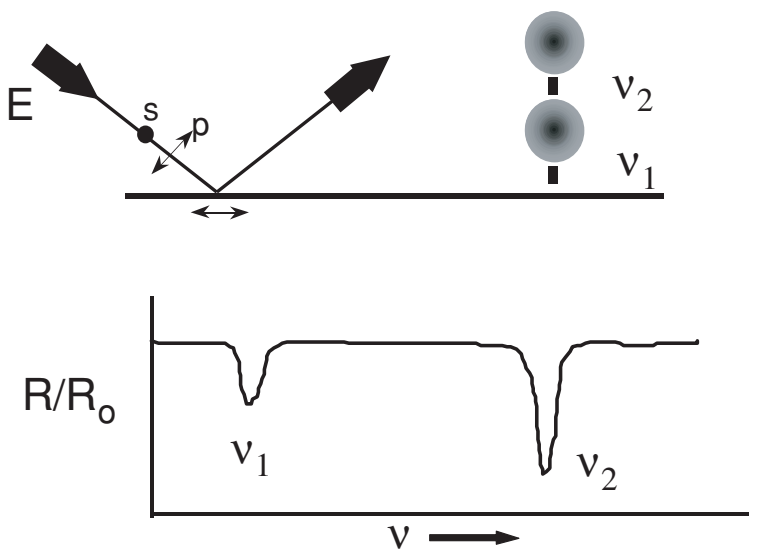

Figure 2. Schematic of a surface infrared reflection-absorption experiment typically used to study adsorbates on a metal surface. In the lower part of the figure we show the changes expected to be induced in the reflection spectrum by the adsorbate. For diatomic species the intramolecular modes $\left(v_{2}\right)$ are usually at higher frequencies and stronger than the extra-molecular or bonding modes $\left(v_{1}\right)$. Note that in this simple picture, no change in reflection is expected except in the vicinity of the vibrational modes.

sputtering with $\mathrm{Ne}$ at $500 \mathrm{~V}$ and a current of $5 \mu \mathrm{A}$ were needed altogether in order to clean each sample for the first time after baking. After the first $20 \mathrm{~min}$ of this process, the film resistance reached a minimum, but Auger spectra showed carbon contamination of the surface. After each of the experiments that followed, typically 10 min of ion bombardment $\left(\mathrm{Ne}^{+}, 500\right.$ $\mathrm{eV}, 5 \mu \mathrm{A}$ ) were needed to clean the sample.

The loss in film thickness due to sputtering was compensated by evaporating additional copper at room temperature until the initial resistance of the film was retrieved. The regrowth process was followed by a short annealing at $400 \mathrm{~K}$. The LEED pattern confirmed that the films were again of (1 11 )-orientation. The subsequent cooling down of the sample to $80 \mathrm{~K}$ took approximately $20 \mathrm{~min}$ until the resistance stabilized. The experimental lifetime of the films was limited because the removal of material by sputtering was not completely homogeneous. Repeated recycling of the sample could lead to variations in the film thickness too large to be neglected.

Gas dosing for the experiments described here was accomplished by backfilling the chamber with the gas of interest. To determine the coverage we used techniques of temperatureprogrammed desorption, LEED and coverage-dependent spectra.

\section{Theoretical discussion}

\subsection{Introduction and motivation}

The evolution of the experimental programs has involved much interaction with the theory developed by Persson's group. Therefore, it is useful to begin by reporting the findings of the early experiments which motivated this theoretical development, followed by the theory itself, and then the additional experiments motivated by this theory.

To help understand the early data, we show the experiment schematically in figure 2. An infrared beam reflects at grazing incidence from a metal surface with a low coverage of diatomic molecules. For this experiment, one would intuitively expect the changes in 


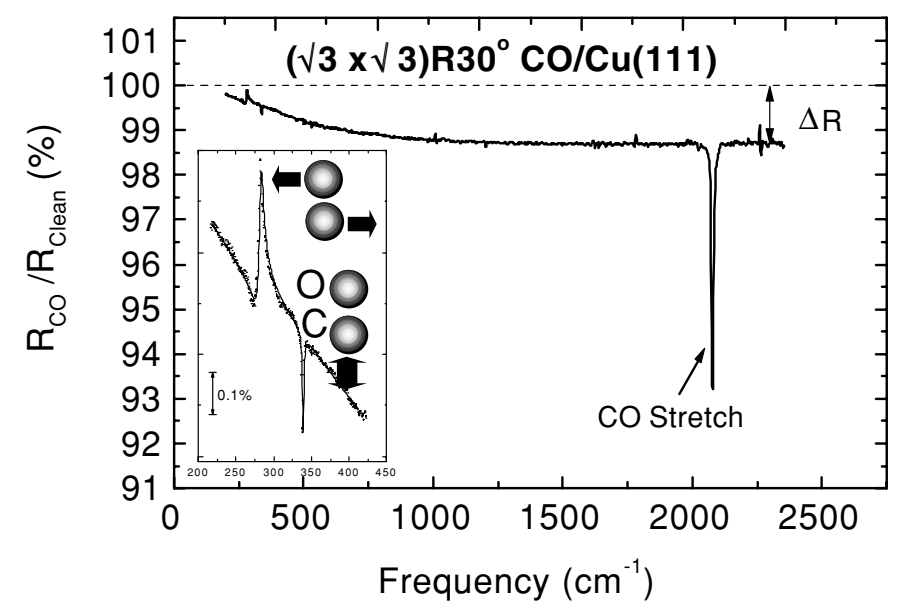

Figure 3. Measured reflectance changes induced by a third of a monolayer of $\mathrm{CO}$ on a $\mathrm{Cu}\left(\begin{array}{lll}1 & 1 & 1\end{array}\right)$ surface shown on an absolute scale. The most noticeable feature is the CO stretch at $\sim 2100 \mathrm{~cm}^{-1}$. The spectrum also contains several spikes which are due to characteristics of the source. The low frequency measurements are shown in the inset on a larger scale together with the assigned vibrational modes. Note that there are broadband changes and an anti-absorption peak at $275 \mathrm{~cm}^{-1}$. These unexpected features provide a powerful insight into the dynamics when combined with the theoretical models.

reflectance induced by the adsorbate to show two loss features, one due to the interatomic stretch mode and the other due to the molecule-surface mode. Any other modes parallel to the surface are not expected to play a role since there is no parallel electric field above the metal surface. Figure 3 shows what actually happens on an absolute scale for $\mathrm{CO} / \mathrm{Cu}$ [14]. Here we have plotted the changes in the reflectance induced by the $\mathrm{CO}$ sub-monolayer. The low frequency data are shown on an expanded scale in the inset. The main feature of this spectrum is a large dip in reflectivity due to the well-known intramolecular vibrational mode at $2100 \mathrm{~cm}^{-1}$. However, the spectrum also shows spikes which are due to noise from the synchrotron source. These features could readily be identified by changing the velocity of the scanning mirror of the Michelson interferometer, and hence distinguished from the features shown in the inset of figure 3, namely the carbon-metal bonding vibrational mode at $340 \mathrm{~cm}^{-1}$. Two other important features are to be noted, namely a broadband reflectance change and a peak at $275 \mathrm{~cm}^{-1}$.

Due to the high intrinsic resolution of IRAS compared with electron energy loss spectroscopy $\left(1 \mathrm{~cm}^{-1}\right.$ compared with $\left.40 \mathrm{~cm}^{-1}\right)$, isotopic substitution can be used to identify modes. In this case with two unknowns two substitutions needed to be made. However, we actually measured all four isotopes so that, in conjunction with helium atom scattering, the

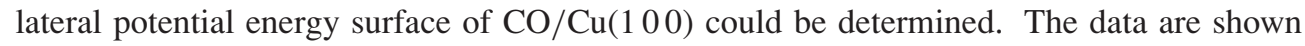
in figure 4 [16]. From these data the 'antiabsorption' peak was found to be the frustrated rotational mode. This was particularly surprising, since in the traditional understanding of IRAS, modes parallel to the surface are called dipole-forbidden, because no external field can be created to excite them. They are therefore not expected to be observable.

\subsection{Theory}

The rather surprising observations reported above need to be explained. They are important since the energy and phase relaxation of vibrational excitations of molecules and atoms 

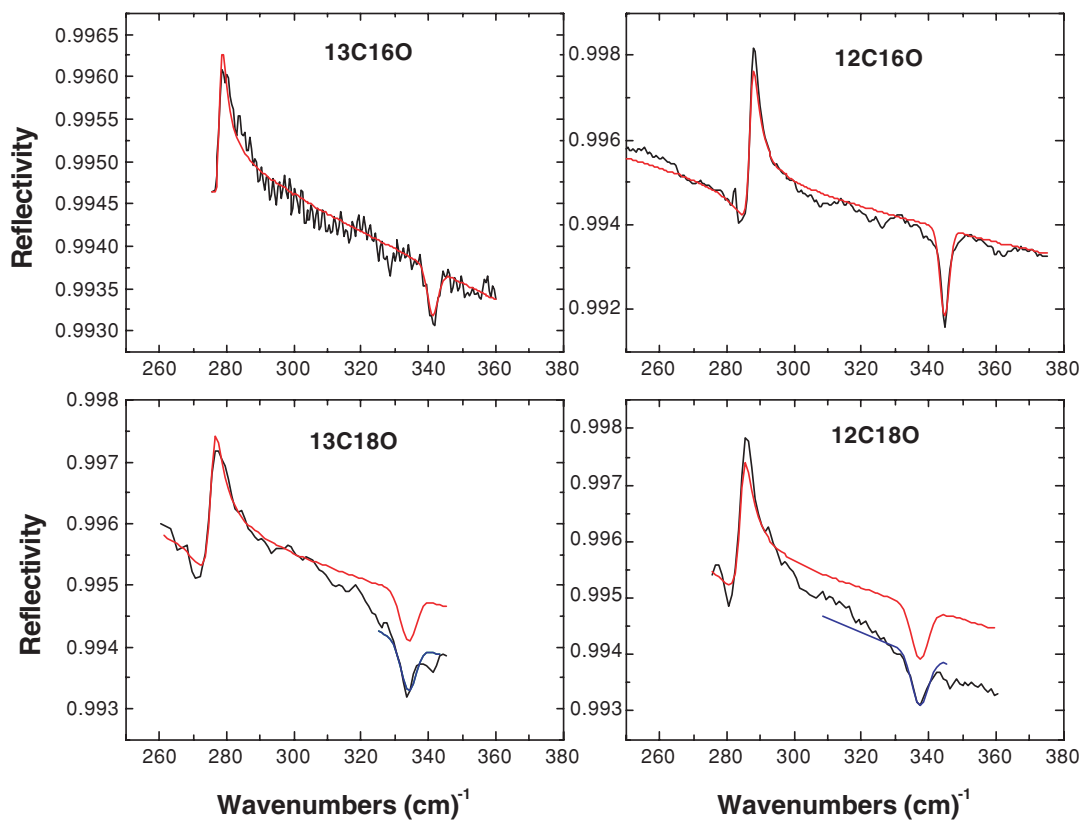

Figure 4. Isotope dependences of the low frequency reflectance changes induced by half a monolayer of $\mathrm{CO} / \mathrm{Cu}\left(\begin{array}{lll}1 & 0 & 0\end{array}\right)$.

adsorbed on solid surfaces contain important information about the couplings of the adsorbates with each other and with their surroundings. The relative importance of vibrational energy in interfacial reactions depends on the rate at which it equilibrates with the surface compared to the reaction rate. A rapid transfer of the adsorbate vibrational energy to the substrate simply leads to an increase in temperature of the solid, but a slow decay of vibrational excitations becomes important in surface reactions.

An extensive theoretical treatment of this problem has been made by Persson [17], in which he uses a free-electron approach to link the reflectivity to other macroscopic effects such as surface friction and electronic lifetime, via intrinsic properties of the substrate such as the plasma frequency, the mean-free path and the skin depth. The problem is complicated by the fact that the wavelengths used are of a scale which bridges what are known as the local and non-local field approximations and which refers to whether the electron experiences changes in electric field as it moves between collisions. The theory not only explains the broadband reflectivity changes and the anti-absorption mode but also links them to resistivity measurements as a method of deepening the understanding of these effects. The basic model as it applies here involves strong non-adiabatic vibration/electron couplings. In $\mathrm{Cu}$ the meanfree path of the electrons at $3000 \AA$ is longer than the penetration depth of $300 \AA$, and the electrons in the substrate are excited by the incoming field. The unexpected features are a manifestation of a breakdown of the Born-Oppenheimer rule (the electrons do not follow the ionic vibrational motion) and are due to scattering of substrate electrons off the adsorbates.

For simplicity in presentation here we use a local field approach, which qualitatively gives the same results as the full quantitative treatment. The reflectivity change induced by an adsorbate can be calculated from the equations of motion. The friction between the adsorbate and the 'Drude-electrons' is assumed to depend only on the relative motion between the adsorbate (velocity $\mathrm{d} u / \mathrm{d} t$ ) and the 'hydrodynamic' drift velocity of the electrons right below 
the interface (velocity $v_{\|}$parallel to the interface). The force $f$ between the electrons and the adsorbate $($ action $=$ reaction $)$ at an ideally smooth 'jellium surface' is expressed as:

$$
f=-M \eta\left(\frac{\mathrm{d} u}{\mathrm{~d} t}-v_{\|}\right)
$$

with $\eta$ being the friction coefficient.

Persson and Volokitin do not define the exact meaning of $M$, but it may be assumed to be the mass of a single particle moving parallel to the surface, or it may signify a kind of inertia for an adsorbed molecule performing a frustrated rotation around an axis parallel to the surface. This definition has the advantage that, within the approximations of the model, the lifetime $\tau$ of the frustrated translation of an adsorbate is given by

$$
\frac{1}{\tau}=\frac{1}{\tau_{e-h}}+\frac{1}{\tau_{\text {phonons }}} \quad \text { with } \quad \eta=1 / \tau_{e-h} .
$$

If the adsorbate has a natural oscillation frequency of $\omega_{o}$, then one can write an equation of motion as follows:

$$
M \ddot{u}+M \omega_{o}^{2} u+M \eta\left(\dot{u}-v_{\|}\right)=0 .
$$

If $v_{\|} \approx \exp (-\mathrm{i} \omega t)$, then it follows that:

$$
v_{\|}-\dot{u}=\frac{\omega^{2}-\omega_{o}^{2}}{\omega^{2}-\omega_{o}^{2}+\mathrm{i} \omega \eta} v_{\|} .
$$

The time averaged adsorbate-induced power is, therefore

$$
P=N\left\langle f \cdot\left(v_{\|}-\dot{u}\right)\right\rangle=N M \eta\left\langle v_{\|}^{2}\right\rangle h(\omega) / n^{2} e^{2}
$$

where

$$
h(\omega)=\frac{\left(\omega^{2}-\omega_{o}^{2}\right)^{2}}{\left(\omega^{2}-\omega_{o}^{2}\right)^{2}+\omega^{2} \eta^{2}}
$$

\langle\rangle is an average over time and $N$ is the number of adsorbates. Now

$$
\Delta R=-P / I_{o} A \quad \text { and } \quad I_{o}=c E_{o}^{2} / 8 \pi
$$

so that

$$
\Delta R=\frac{8 \pi}{c} \frac{n_{a}}{\cos \theta} \frac{M \eta}{n^{2} e^{2}} \frac{\left\langle J_{\|}^{2}\right\rangle}{E_{o}^{2}} h(\omega) .
$$

But $\tau$ and the mean free path are long, so

$$
\left\langle J_{\|}^{2}\right\rangle=\omega_{p}^{2} E_{o}^{2} / 8 \pi
$$

and the reflectivity change is

$$
\Delta R=-\frac{4}{c} \frac{n_{a}}{n} \frac{M}{m} \frac{\eta}{\cos \theta} h(\omega)
$$

where $m$ is the free electron mass and $\theta$ is the angle of incidence.

At the very grazing incidence angles usually used for the IRAS experiments, this expression should be multiplied by a further factor $F[15]$.

$$
F=\frac{\cos ^{2} \theta}{\cos ^{2} \theta+\omega^{2} / \omega_{p}^{2}} .
$$

Note that the above expression still has unknown quantities, namely $M$ and $\eta$. It only applies to high frequencies for which $\omega \gg 1 / \tau_{B}$ and $\omega / \omega_{p} \gg v_{F} / c$. One thing not described 


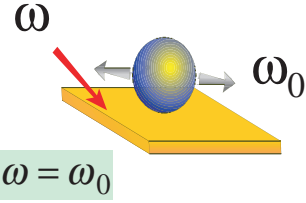

No Friction!!

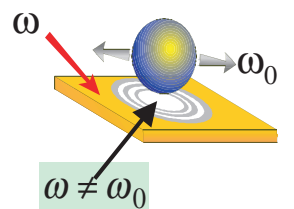

Friction

Figure 5. Sketch to illustrate the concept of atomic friction between an adsorbate and the electrons in the substrate. The substrate electrons are being driven by an external field at a frequency $\omega$ while the adsorbate vibrates at a natural frequency of $\omega_{0}$. When the two frequencies match, there is no relative motion between the adsorbate and the electrons and this friction term vanishes.

by this is the exact nature of the frequency dependence of the background change, which starts from zero at zero frequency up to the asymptotic limit which is described above. The full calculation is in [17] and explains the background absorption extremely well as can be seen from the fits in figure 4. One can also see from equation (9), how the adsorbate parallel mode gives a maximum at $\omega=\omega_{0}$. Qualitatively it is understood with reference to figure 5 . The background absorption is caused by scattering (friction) of conduction electrons by the adsorbate, and when the adsorbate is moving at the same frequency as the electrons, this friction disappears.

A deeper analysis of the Persson [17] model can be made by looking at the equations of motion of the electrons in the substrate which are driven by the incident electric field

$$
\ddot{x}+\frac{1}{\tau_{B}} x+\frac{M n_{a}}{m n d \tau_{e-h}}(\dot{x}-\dot{q})=\frac{e}{m} E .
$$

Putting $E=E(\omega) \exp (-\mathrm{i} \omega t)$ we obtain

$$
\begin{aligned}
& \left(-\omega^{2}+\Omega^{2}-\mathrm{i} \frac{\omega}{\tau_{e-h}}\right) q(\omega)=-\mathrm{i} \frac{\omega}{\tau_{e-h}} x(\omega) \\
& \left(-\omega^{2}-\mathrm{i} \frac{\omega}{\tau_{B}}\right) x(\omega)-\mathrm{i} \omega \frac{M n_{a}}{m n d \tau_{e-h}}(x(\omega)-q(\omega))=\frac{e}{m} E(\omega) .
\end{aligned}
$$

Defining the conductivity $\sigma(\omega)$ as

$$
\begin{aligned}
& -\mathrm{i} w \operatorname{ex}(\omega)=\sigma(\omega) E(\omega) \\
& \sigma(\omega)=\frac{n e^{2}}{m}\left[-\mathrm{i} \omega+\frac{1}{\tau_{B}}+\frac{M n_{a}}{m n d \tau_{e-h}} \frac{\Omega^{2}-\omega^{2}}{\Omega^{2}-\omega^{2}-\mathrm{i} \omega / \tau_{e-h}}\right]^{-1} .
\end{aligned}
$$

If we compare this with the usual formula,

$$
\sigma(\omega)=\frac{n e^{2}}{m} \frac{1}{-\mathrm{i} \omega+1 / \tau} .
$$

Setting $\omega \gg 1 / \tau_{B}$, we obtain the following expression for the change $\Delta \rho$ of the resistivity of a thin film of thickness $d$, covered by $n_{a}$ adsorbed molecules per $\mathrm{cm}^{2}$

$$
\Delta \rho=\frac{M n_{a}}{n^{2} e^{2} d \tau_{e-h}}
$$

where $n$ is the free electron density. Notice that $\Delta \rho$ is independent of the initial resistivity $\rho$ of the film. 
A comparison of equations (13) and (21) leads to a ratio between the change of reflectance and the change of resistance which, remarkably, is given as

$$
\frac{\Delta R}{\Delta W}=-\frac{\omega_{p}^{2} d^{2}}{\pi c} \frac{F}{\cos \theta}
$$

with $d \Delta W=\Delta \rho$ and $\omega_{p}^{2}=4 \pi n e^{2} / m$ (plasma frequency). Significantly, this ratio does not depend on $n_{a}, M$ and $\eta$, and is therefore independent of the state of adsorption (bonding, concentration, 'mass') of the species. Experimental confirmation of the validity of this ratio is a powerful test of the atomic scale friction model in explaining the surface dynamics of these systems.

Relaxation effects can be calculated from the data in two ways. First, the initial slope of $\Delta W / \Delta n_{a}$ is system specific. In earlier papers, Persson [18] suggested that the initial slope $\left(\partial_{\rho} / \partial n_{a}\right)_{n_{a} \rightarrow 0}$ of the resistivity is related to the friction coefficient by

$$
\eta=\left.\frac{n^{2} e^{2}}{M} d \frac{\partial \rho}{\partial n_{a}}\right|_{n_{a} \rightarrow 0} .
$$

Another handle on $\eta$ may be obtained from the microscopic theory of the friction coefficient based on the Newns-Anderson model [18, 19]:

$$
\eta=\frac{\hbar}{M} \Gamma \rho\left(E_{F}\right)\left\langle k_{p}^{2}\right\rangle
$$

where $\Gamma$ is the width of the resonance state induced by the absorbate and $\rho(E)$ the induced density of states and

$$
\left\langle k_{p}^{2}\right\rangle=\frac{\sum_{k} k_{p}^{2}\left|V_{a k}\right|^{2} \delta\left(E_{F}-\varepsilon_{k}\right)}{\sum_{k}\left|V_{a k}\right|^{2} \delta\left(E_{F}-\varepsilon_{k}\right)}
$$

where the electron transfer interaction between affinity level $|a\rangle$ and metal state $|k\rangle$ (component parallel to the surface $k_{p}$ ) is given by $V_{a k}$.

This yields

$$
\Delta W=\frac{\hbar n_{a}}{(\text { ned })^{2}} \Gamma \rho\left(E_{F}\right)\left\langle k_{p}^{2}\right\rangle .
$$

Note that equation (26) does not contain $M$ either. This demonstrates that the theory describes a purely electronic effect, namely the resistance induced by an adsorbate acting as a surface point defect, whose vibrations are not excited. Thus the adsorbate acts as a static defect. Therefore, equation (26) has much in common with the scattering by transition metal impurities in a bulk free electron metal. Of course the experimental data will also contain contributions due to inelastic scattering, for instance, the creation and annihilation of quanta of the frustrated translation of the adsorbates. This is not included in Persson's theory yet.

One further quantity that one can obtain from these data is the scattering cross section, $\sigma$, which is defined as

$$
\sigma=\frac{1-p}{n_{a}}
$$

where $p$ is the specular scattering probability. Persson derived the relationship between the damping rate $\eta$, and the scattering probability thus,

$$
\eta=(1-p) \frac{16 n m v_{F}}{3 n_{a} M}
$$

where $v_{F}$ is the Fermi velocity. 


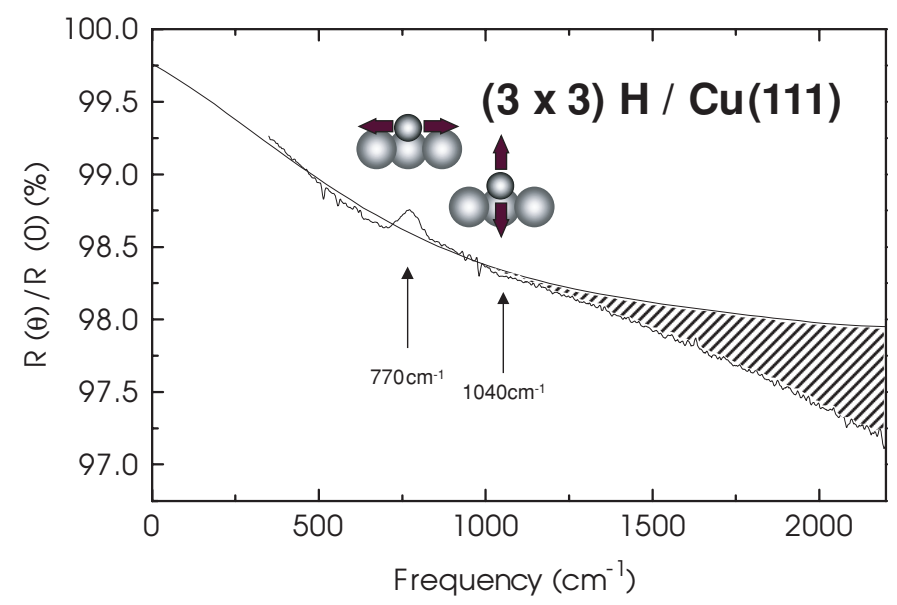

Figure 6. Measured changes in reflectance induced by a sub-monolayer of hydrogen adsorbed on a $\mathrm{Cu}\left(\begin{array}{lll}1 & 1 & 1\end{array}\right)$ surface. The shaded area is the fit to the simple theory given by equation (13). The discrepancies are discussed in [20], but the main point is the background change and the observation of the parallel vibrational mode.

\section{Experiments and discussion}

\subsection{Atomic adsorbates on Cu single crystals}

A good example of the rather broad application of the above theory is shown for the simpler system of atomic $\mathrm{H} / \mathrm{Cu}\left(\begin{array}{lll}1 & 1 & 1\end{array}\right)$ [20]. The system and its interpretation within a simple extension to the theoretical framework above, provide an example of the strength of the technique in elucidating dynamics.

In figure 6 we show the IR response of this system, and again see both the background change and the frustrated translational mode. For this system there is only a hint of the $\mathrm{H}-\mathrm{Cu}$ stretch.

Again, isotopic substitution confirmed the assignments. Due to the fact that $\mathrm{H}$ on $\mathrm{Cu}$ may experience additional damping due to lateral tunnelling of the adsorbates or dephasing, we may enhance the theory to add an additional term $f_{2}=-M \gamma \dot{\boldsymbol{u}}$ in equation (6). Then we obtain

$$
\Delta R=-\frac{4}{c} \frac{n_{a}}{n} \frac{M}{m} \frac{\eta}{\cos \theta}\left(1-\frac{\omega^{2} \eta(\eta+\gamma)}{\left(\omega^{2}-\omega_{o}^{2}\right)^{2}+\omega^{2}(\eta+\gamma)^{2}}\right) .
$$

The anti-absorption peak has a width $\Gamma=\eta+\gamma$ and the area under the peak is independent of $\gamma$. When $\gamma=0$ the reflectivity at resonance returns to the reflectivity of the clean surface (i.e. $\Delta R=0$ for $\left.\omega=\omega_{o}\right)$. When $\gamma$ is non-zero, $\Delta R$ at resonance is a fraction $\gamma /(\eta+\gamma)$ of its off-resonance value (figure 7). Let us apply the results above to the $\mathrm{H} / \mathrm{Cu}\left(\begin{array}{lll}1 & 1 & 1\end{array}\right)$ system. From figure 6 , the linewidth of the anti-absorption peak is $\Gamma \approx 62 \mathrm{~cm}^{-1}$ and $\eta /(\gamma+\eta) \approx 0.12$. Hence, $\eta \approx 7.5 \mathrm{~cm}^{-1}$ and $\gamma \approx 54.5 \mathrm{~cm}^{-1}$. An electronic friction $\eta \approx 7.5 \mathrm{~cm}^{-1}$ corresponds to a lifetime $\tau=0.7 \mathrm{ps}$. This value is very reasonable. For example, for hydrogen on Ni(l 111$)$ from surface resistivity measurements one can deduce the lifetime $\tau \sim 1 \mathrm{ps}$. For deuterium it was found that $\Gamma \approx 52 \mathrm{~cm}^{-1}$ and $\eta /(\gamma+\eta) \approx 0.11$. Thus, $\eta \approx 6 \mathrm{~cm}^{-1}$ and $\gamma \approx 46 \mathrm{~cm}^{-1}$ and the corresponding lifetime was 0.9 ps.

There is a another way of deducing $\eta$ from the data in figure 6 , namely, from the magnitude of the background absorption. Using equation (29) with $n_{a}=0.124 \AA^{-2}$, corresponding to a 


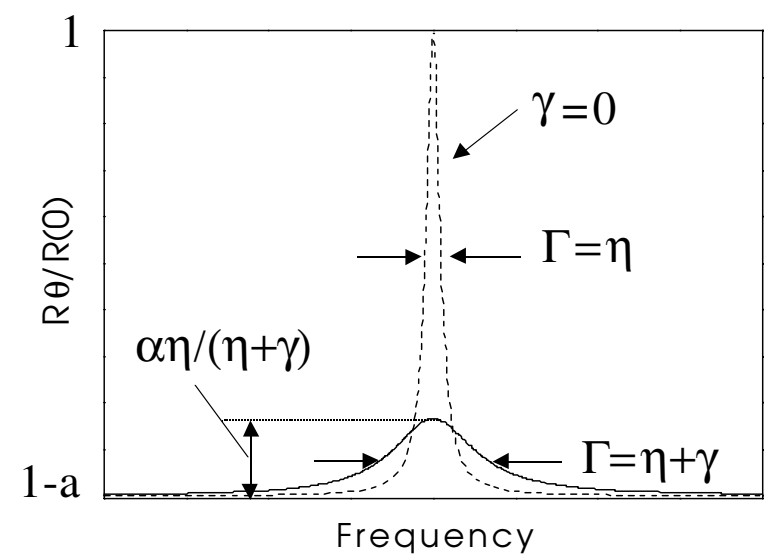

Figure 7. Theoretical shape of the anti-resonance induced by a parallel vibrational mode at a metal surface in the local optics case, i.e. $\omega_{0} \gg v_{F} / \delta$, where $v_{F}$ is the Fermi velocity of the metal, and $\delta=c / \omega_{p}$ is the classical skin depth - that is the ratio between the velocity of light and the plasma frequency. $\eta$ is the surface friction term or the electron-hole pair damping, $\gamma$ is an additional damping term - the 'tunnelling width', and the parameter $\alpha=\left(4 n_{a} M \eta\right) /(\operatorname{cnm} \cos \theta)$.

coverage $\theta=0.7$ (which has been suggested for the $(3 \times 3) \mathrm{H}$ overlayer [21]) gives $\tau \approx 3$ ps or $\eta \approx 1.7 \mathrm{~cm}^{-1}$. This value for $\tau$ is about a factor of four longer than that deduced from the area under the anti-absorption peak. This result is, however, expected because for an ordered adsorbate structure, the damping $\eta$, deduced from the IRAS background absorption, corresponds to the energy relaxation time of the collective mode, where all the adsorbates oscillate in phase. This follows from the fact that the photons have practically zero wave vector on the scale of $1 / a$ where $a$ is the adsorbate lattice constant and $\eta$ will be affected by the interference between the electron probability amplitude waves scattered from different adsorbates along the surface. In the dilute limit, however, this interference effect can be neglected and the corresponding $\eta$ will be different. This result is confirmed by thinfilm resistivity measurements [22] for $\mathrm{H}$ on $\mathrm{Ni}\left(\begin{array}{lll}1 & 1 & 1\end{array}\right)$ surfaces, where the adsorbate induced contribution to the film resistivity (per adsorbate) is a factor of four smaller at high coverages. However, the $\eta$ deduced from the anti-absorption resonance when $\gamma \gg \eta$ will approximately correspond to the damping of $\mathrm{H}$ vibrations in the dilute limit. This can be understood as follows. If only inhomogeneous broadening occurred, $\gamma$ would be a measure of the width of a distribution of resonance frequencies. In a more general case where tunnelling and dephasing occur, $\gamma$ corresponds to the imaginary part of a self energy but can still be interpreted as the width of a distribution of resonance frequencies associated with the eigenstates of the system, which because of dephasing will be quite localized. As the photon energy is swept through this band of resonance frequencies, different eigenmodes will be excited, but only a small fraction of the adsorbates will be excited for each frequency. This situation thus corresponds to the dilute limit.

The electronic friction $\eta$ is a crucial input for the discussion of other dynamical processes at surfaces, e.g. surface diffusion. It has been shown [23] that in order to correctly describe the diffusive motion of $\mathrm{H}$ on $\mathrm{Ni}(100)$ an electronic friction in the range $15 \pm 10 \mathrm{~cm}^{-1}$ is necessary. Similarly, from an analysis of neutron scattering data, for tracer diffusion of $\mathrm{H}$ in bulk Pd, it has been deduced [24] that $\eta=25 \pm 10 \mathrm{~cm}^{-1}$. The value we have deduced above from IRAS data, $\eta \approx 10 \mathrm{~cm}^{-1}$, is well in line with these independent data and also in accordance with the first principle calculations [25]. 


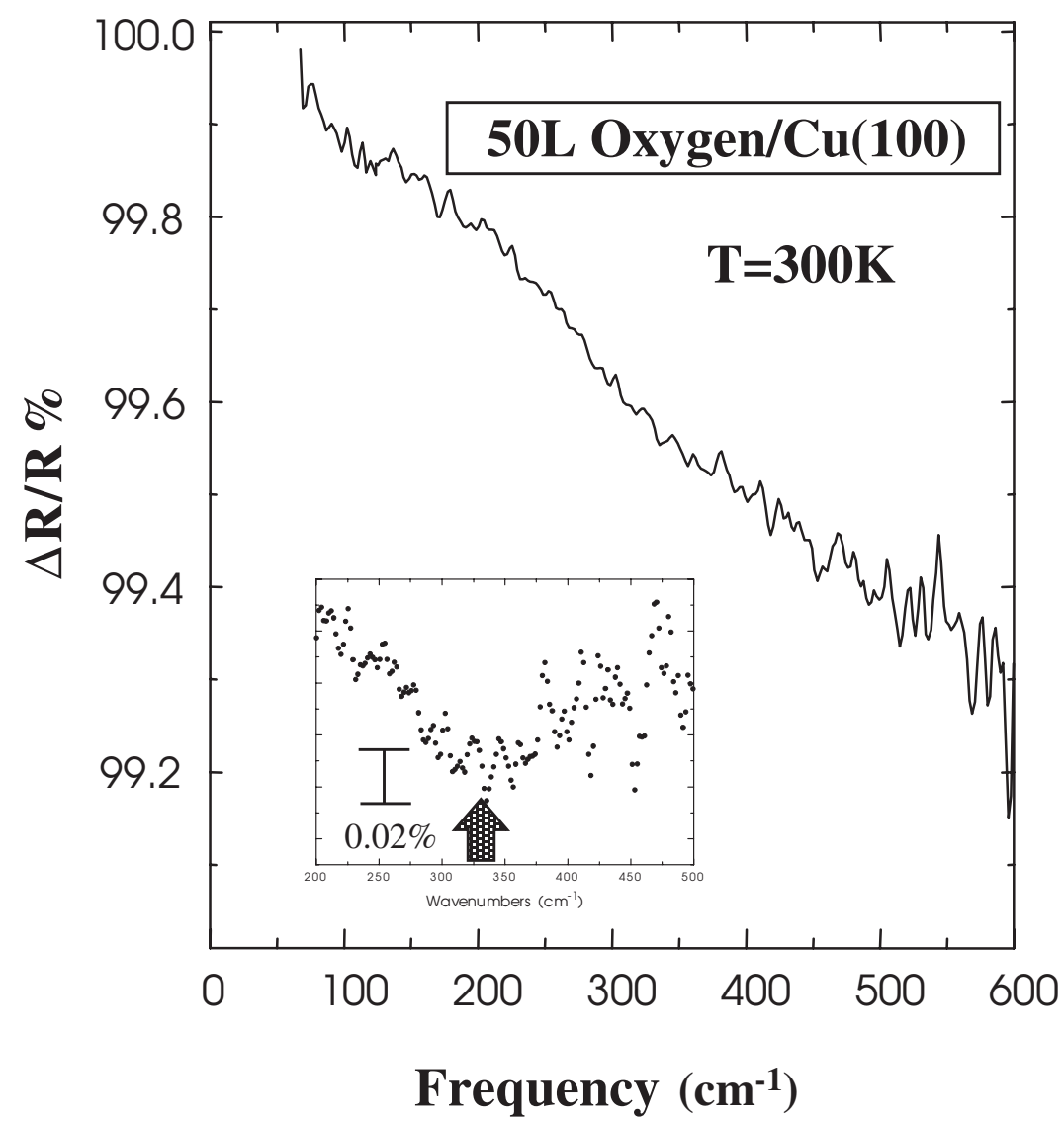

Figure 8. Measured absolute reflectance changes induced by a 50 Langmuir coverage of oxygen on $\mathrm{Cu}(100)$ at $300 \mathrm{~K}$. The broad dip, shown with the background slope removed in the inset, is assigned to the $\mathrm{O}-\mathrm{Cu}$ vibrational mode. Note that the changes are only $\sim 0.03 \%$.

For $\mathrm{O} / \mathrm{Cu}(100)$, figure 8 , the $\mathrm{O}-\mathrm{Cu}$ stretch is very broad and the frustrated translation is too low in frequency to be observable with the present system. Therefore, an analysis as was performed for $\mathrm{H}$ was not possible. However, it is interesting to note that there is an unmistakable background change, showing that the dynamics is similar.

Lastly, we mention some experiments involving alkali metal atoms on surfaces. When $\mathrm{K}$ or $\mathrm{Na}$ was deposited on mechanically polished surfaces of $\mathrm{Cu}$ and $\mathrm{Ag}$, peaks were observed in the IR spectra, see figure 9, which were interpreted as being due to a charge density wave [26]. The peaks attributed to such states, disappeared for electropolished samples, both for $\mathrm{Cu}$ and Ag. From the fits to the data it was possible to gain insight into the surface charge density and dynamics. These experiments illustrate the breadth and diversity or IR studies at surfaces.

\subsection{Molecules on metal single crystals}

In addition to the $\mathrm{CO}$ experiments mentioned earlier, there has been considerable other synchrotron work on molecules on metal single crystals, all of which have studied low frequency modes and the phenomena that cause the background shift. Other experiments 


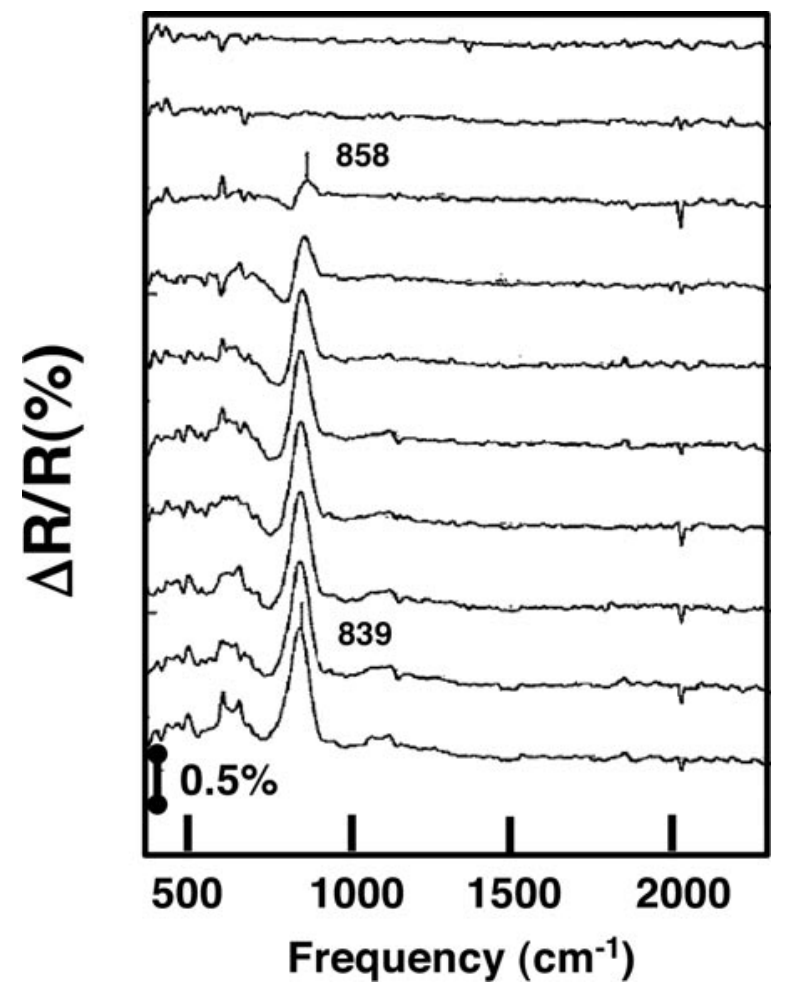

Figure 9. Progressive changes (top to bottom) in the reflectance of a $\mathrm{Cu}\left(\begin{array}{lll}1 & 1 & 1\end{array}\right)$ surface upon increasing deposition of $\mathrm{K}$ at $92 \mathrm{~K}$. The maximum coverage was 1.64 monolayers. The peak is assigned to the development of a charge density wave.

have involved formate [27], and $\mathrm{NO} / \mathrm{Cu}$ [28] as well as $\mathrm{CO}$ and $\mathrm{H}_{2} \mathrm{O}$ [29] on $\mathrm{Ni}$ and $\mathrm{C}_{60}$ on $\mathrm{Cu}$ and $\mathrm{Ag}$ [30].

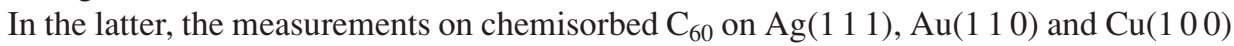
reveal that a non-IR active mode becomes active upon adsorption and that its frequency shifts proportionally with the charge transferred from the metal to the molecule by about $5 \mathrm{~cm}^{-1}$ per electron. The temperature dependence of the frequency and the width of this IR feature has also been followed for $\mathrm{C}_{60} / \mathrm{Cu}(100)$ and was found to agree well with a weak anharmonic coupling (dephasing) to a low frequency mode at $45-75 \mathrm{~cm}^{-1}$, which is possibly the frustrated translational mode of the adsorbed molecules.

It is interesting to see how the large $\mathrm{C}_{60}$ molecule produces a broadband background change similar to atoms and diatomic molecules. The data are shown in figure 10. However, the friction coefficient determined from this particular broadband change results in rather small values $\left(\sim 2 \times 10^{9} \mathrm{~s}^{-1}\right.$ for $\mathrm{Ag}$ and $\mathrm{Au}$, and $\sim 1.6 \times 10^{9} \mathrm{~s}^{-1}$ for $\left.\mathrm{Cu}\right)$, consistent with a marked metallic character of the adsorbed species. In fact, the product $\Gamma \rho\left(E_{F}\right)$ equation (24) is about four times higher for $\mathrm{C}_{60}$ than for $\mathrm{CO}$, and this is largely attributed to the higher value of the adsorbate-induced $\rho\left(E_{F}\right)$.

In addition, pre-dosing of the surfaces with alkali atoms drastically changes the infrared spectra, yielding antiabsorption bands and an increase of the broadband reflectance. These are interpreted as due to strong electron-phonon coupling with induced surface states.

Perhaps the finest experiments to date in terms of reproducibility have been those on $\mathrm{SnCl}_{4}$ and water on thin film carbon and silica surfaces [31] at the synchrotron radiation source (SRS) 


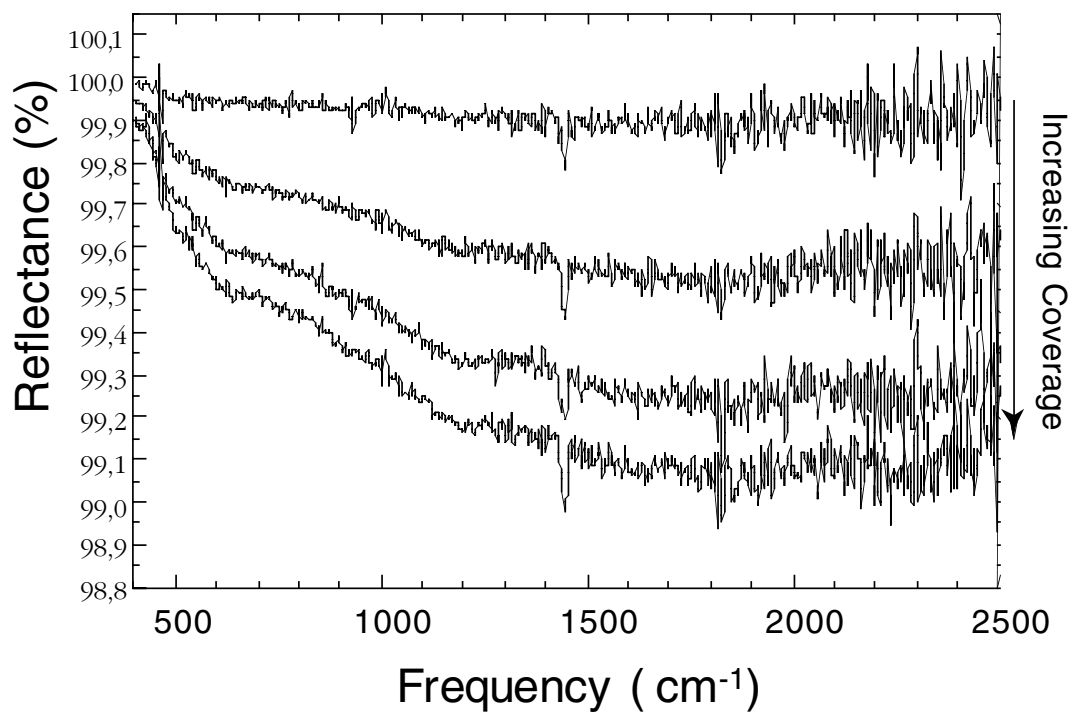

Figure 10. Absolute changes in the reflectance of a $\mathrm{Cu}(100)$ surface upon deposition of $\mathrm{C}_{60}$ at room temperature. Note the broadband change and the absorption band at $1440 \mathrm{~cm}^{-1}$.

at Daresbury in England. These data are of high technological importance involving energysaving coatings on glass. The experiments revealed that for $\mathrm{SnCl}_{4}$ on a thin silica surface at 120 $\mathrm{K}$, in addition to the unperturbed multilayer, there are two highly perturbed $\mathrm{Sn}-\mathrm{Cl}$ stretching modes at $375 \mathrm{~cm}^{-1}$ and $350 \mathrm{~cm}^{-1}$ which indicate the presence of two additional and highly significant surface species. These states could only have been seen due to the reproducibility of $0.0035 \%$ down to $200 \mathrm{~cm}^{-1}$.

\subsection{Thin films}

We now follow up on the theoretical discussion in section 3.2 where it was pointed out that a more rigorous test of the theory would be obtained by measuring simultaneously the reflectance and resistivity changes induced by adsorbate molecules on a thin metal film. If the theoretical understanding is correct, such experiments also allow an understanding of the relaxation parameters independent of many assumptions.

Figure 11 shows the reflectivity changes induced by $\mathrm{CO}$ on thin $\mathrm{Cu}\left(\begin{array}{lll}1 & 1 & 1\end{array}\right)$ films, which are similar to those found for the single crystals, both in terms of the background change and the observation of the frustrated rotational mode seen in the inset. The key result is shown in figure 12. Here we show the simultaneous resistance and reflectance data [15] which show a linear relationship - a beautiful confirmation of the two aspects of the theory presented above.

Relaxation effects for these films can be quantified by referring back to equation (23). For the $42 \mathrm{~nm}$ film, we found $\left(\partial W / \partial n_{a}\right)_{n_{a} \rightarrow 0}=2.45 \times 10^{10} \mu \Omega \AA^{2}$ and $d\left(\partial \rho / \partial n_{a}\right)_{n_{a} \rightarrow 0}=$ $4.33 \times 10^{2} \mu \Omega \mathrm{cm} \AA^{3}$, yielding $\eta=1.73 \times 10^{10} \mathrm{~s}^{-1}$. As discussed above, this value may characterize $\mathrm{CO}$ bound at defect sites (isolated molecules) or $\mathrm{CO}$ molecules with a lower occupation of the $2 \pi^{*}$ orbital.

$\eta$ may also be derived from the asymptotic limit of the reflectance change via equation (13). From a coverage of $\approx 0.4$, and using $n_{a}=0.061 \AA^{-2}, M=28 \times 1.66 \times 10^{-27} \mathrm{~kg}, n=$ $8.47 \times 10^{-2} \AA^{-3}$ and $\Delta R=1.15 \%$ follows $\eta=1.23 \times 10^{10} \mathrm{~s}^{-1}$. The use of equation (4) sets a lower limit for the friction coefficient, as the factor $\cos ^{-1} \theta$ has to be corrected. 


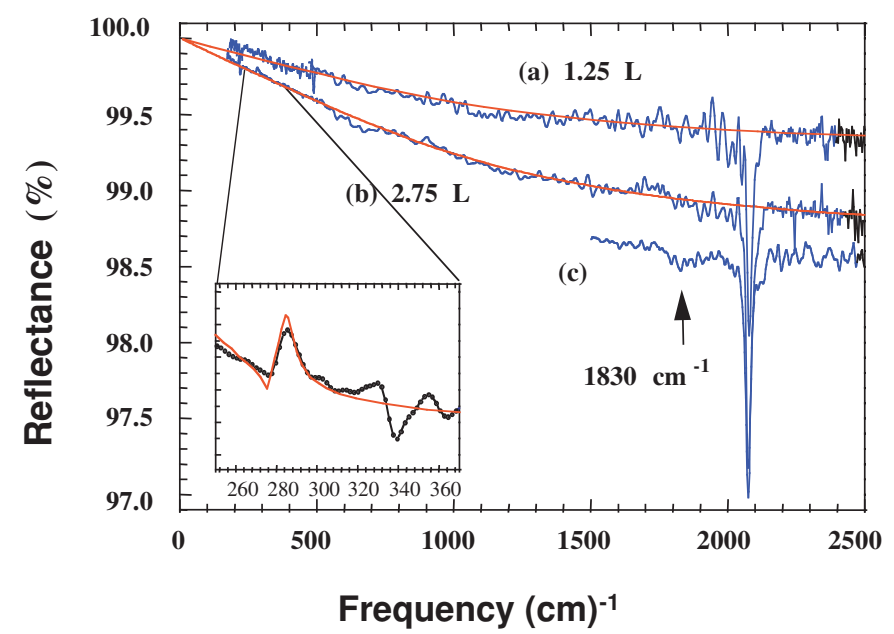

Figure 11. Absolute reflectance changes induced by $\mathrm{CO}$ on a $67 \mathrm{~nm}$ film of $\mathrm{Cu}(111$ orientation). Curve (c) is the result of co-adding several scans and reveals the intramolecular mode at bridge sites. The inset is an enlargement of the low frequency region for a $75 \mathrm{~nm}$ film. The solid lines are fits to the data using the theory discussed in the text.

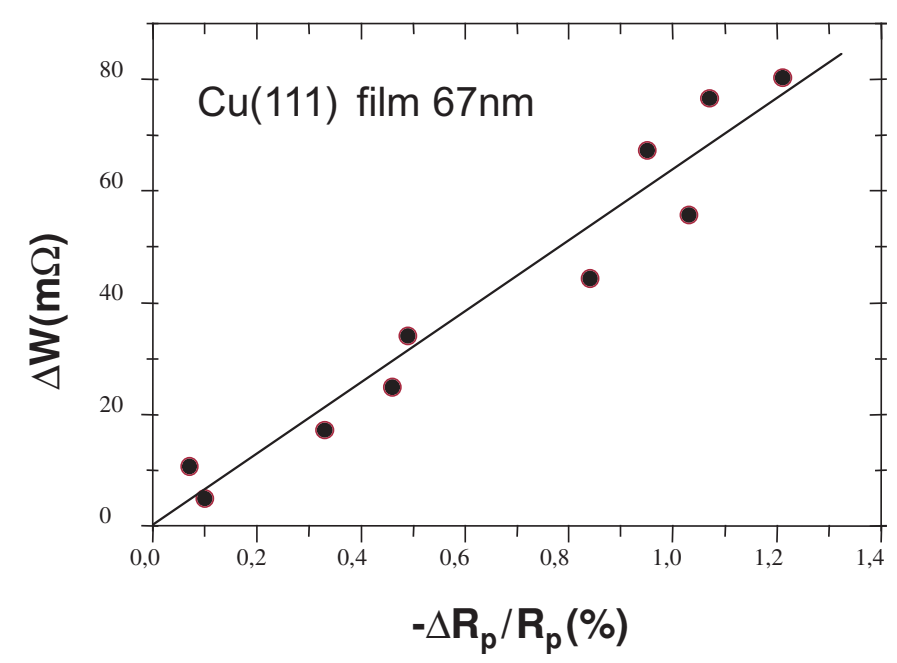

Figure 12. The verification of equation (23) confirmed by the measured linear dependence of the dc resistance change on the reflectance change for a $67 \mathrm{~nm} \mathrm{Cu}\left(\begin{array}{lll}1 & 1 & 1\end{array}\right)$ film dosed with $\mathrm{CO}$.

In view of the difficulties in obtaining a precise value of the film thickness, and of taking into account the factor $F$, this free electron model appears to give striking agreement with many of the experiments. However, it is also fair to say that some discrepancies have been found, the first notable one being that for formate [32] on $\mathrm{Cu}(100)$, which has no reflectance change despite a resistance change. Tobin's group has made extensive investigations into these issues using a globar source and a fixed frequency of $2750 \mathrm{~cm}^{-1}$. They have studied epitaxially grown thin films, and analysed both reflectance and resistivity changes for different films and adsorbates. Not all the systems follow the behaviour expected from the theory in equation (22), which in addition to the linearity prediction, also implies that the ratio of the reflectance 


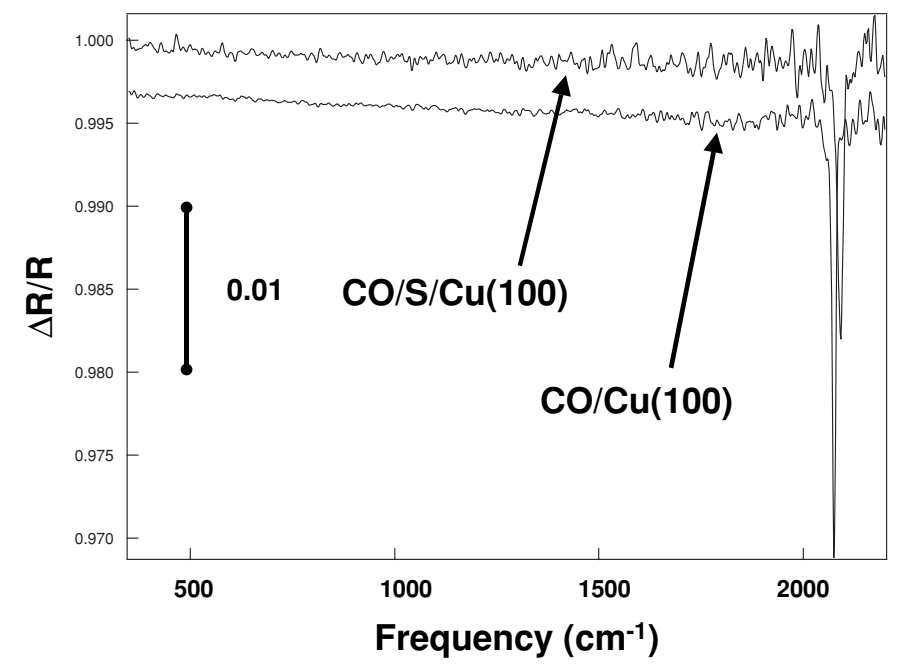

Figure 13. Co-adsorbed systems - absolute changes in the reflectance of $\mathrm{CO} / \mathrm{Cu}(100)$ and $\mathrm{CO}$ on sulfur pre-dosed $\mathrm{Cu}\left(\begin{array}{ll}1 & 0\end{array}\right)$. Note the smaller change induced by the $\mathrm{CO}$ in the latter case.

change and reflectivity depends only on the properties of the substrate. They have studied the discrepancies in some detail, and the investigation currently presents one of the forefront challenges to the theory.

\subsection{Scattering cross sections}

As stated in section 3.2 and specified in equations (27) and (28), some insight can be obtained into adsorbate scattering cross sections. Thus, Kuhl et al [33] used the relationships to determine that for $\mathrm{CO} / \operatorname{Pt}(111), \sigma=0.8 \AA^{2}$ for coverages below 0.33 monolayers. For comparison the area per Pt atom on the surface is $6.6 \AA^{2}$ and the area per CO molecule at 0.5 monolayer coverage is $13.2 \AA^{2}$ so that $\mathrm{CO}$ is a rather weak scatterer.

An analysis in terms of scattering cross sections was taken a step further by Hirschmugl who studied co-adsorbed systems [34]. Specifically, some very interesting behaviour was found for $\mathrm{S}$ co-adsorbed with $\mathrm{CO}$ on $\mathrm{Cu}(100)$, in which the dynamics, or energy transfer between substrate and adsorbates, was dominated by scattering from the $\mathrm{S}$. The data are shown in figure 13, and yield the interesting result that $\mathrm{CO}$ modifies the reflectance of a bare

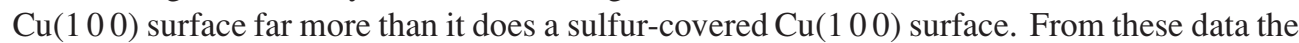
cross sections are estimated to be $34 \AA^{2}$ for S at defect and step edges, but less than $10 \%$ of this value for terrace sites, while the cross section for $\mathrm{CO}$ was found to be $4.2 \AA^{2}$, independent of whether $\mathrm{S}$ was present. This is possible because the scattering cross sections are derived from the slopes of the plots of asymptotic background change as a function of coverage, and these are similar for $\mathrm{CO} /\left(\mathrm{S} / \mathrm{Cu}\left(\begin{array}{lll}1 & 0 & 0\end{array}\right)\right)$ and $\mathrm{CO} / \mathrm{Cu}(100)$. Clearly, these initial experiments point to a new potential of surface IR spectroscopy which will give interesting pointers into catalytic poisons, as well as surface friction modification.

\subsection{Surface reactions}

In addition to the simple adsorbate/metal measurements, some work has ensued in the area of surface reactions. Thus, Hoffmann and collaborators used the far-IR capabilities of the 


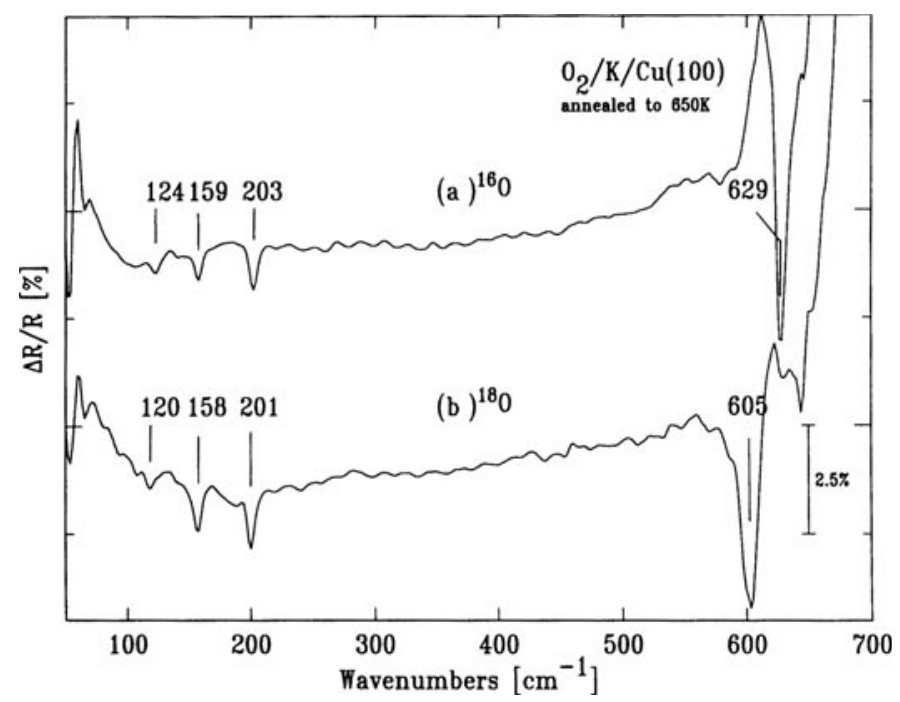

Figure 14. Grazing incidence reflectance spectra at low frequencies for a K-assisted oxidation of

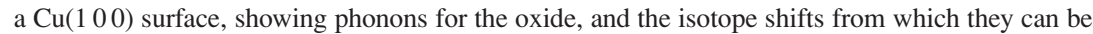
identified. Plotted are the changes induced to the clean surface by the oxidation process.

synchrotron to study the alkali-promoted oxidation of $\mathrm{Cu}$ [35]. They used polyethylene windows and measured with good signal-to-noise down below $100 \mathrm{~cm}^{-1}$. Figure 14 shows spectra obtained after annealing a $\mathrm{K}$ - and $\mathrm{O}_{2}$-covered $\mathrm{Cu}$ surface at $650 \mathrm{~K}$ to form the potassium superoxide.

\subsection{Adsorbate-adsorbate coupling}

As the density of adsorbates builds up on a surface, coupling interactions can occur between adjacent dipoles. In addition there are also chemical shifts. Hirschmugl and Williams [36] showed that measurements could be made of isotopic mixtures by backfilling the chamber with both gases at once while monitoring the ratio with a residual gas analyser. An example for $\mathrm{Cu}\left(\begin{array}{lll}1 & 0 & 0\end{array}\right)$ is shown in figure 15. Mixed isotope measurements at low frequencies have been reported by Wang et al [37], but this is the first work aimed specifically at studying chemical and coupling effects for molecules on surfaces. Previous work for $\mathrm{CO}$ on $\mathrm{Cu}$ was focused entirely on the intra-molecular $\mathrm{C}-\mathrm{O}$ stretch [38-40]. In the discussion that follows, positive shifts are defined as those to higher frequencies with increasing coverage.

Mixed isotope data allow one to separate and determine chemical and coupling effects as they affect vibrational modes of adsorbates on surfaces. Specifically, by diluting CO in a matrix of ${ }^{13} \mathrm{C}^{18} \mathrm{O}$, one essentially removes all vibrational coupling terms. In detail, then the analysis goes as follows. First we define three limits as illustrated in figure 16 . Thus the singleton frequency is the value for an isolated adsorbate as shown in figure 16(a). The dilution limit frequency refers to the value of a mode for a trace of one isotope immersed in a full or ordered overlayer coverage of the other isotope as illustrated in figure 16(b). Finally, the homogeneous limit frequency is obtained for the same full, or ordered overlayer coverage, but of only one species as illustrated in figure 16(c). From these the chemical and coupling terms can be extracted. The chemical shift is the difference between the dilution limit frequency and the singleton frequency. The coupling shift can be determined from the direction and 


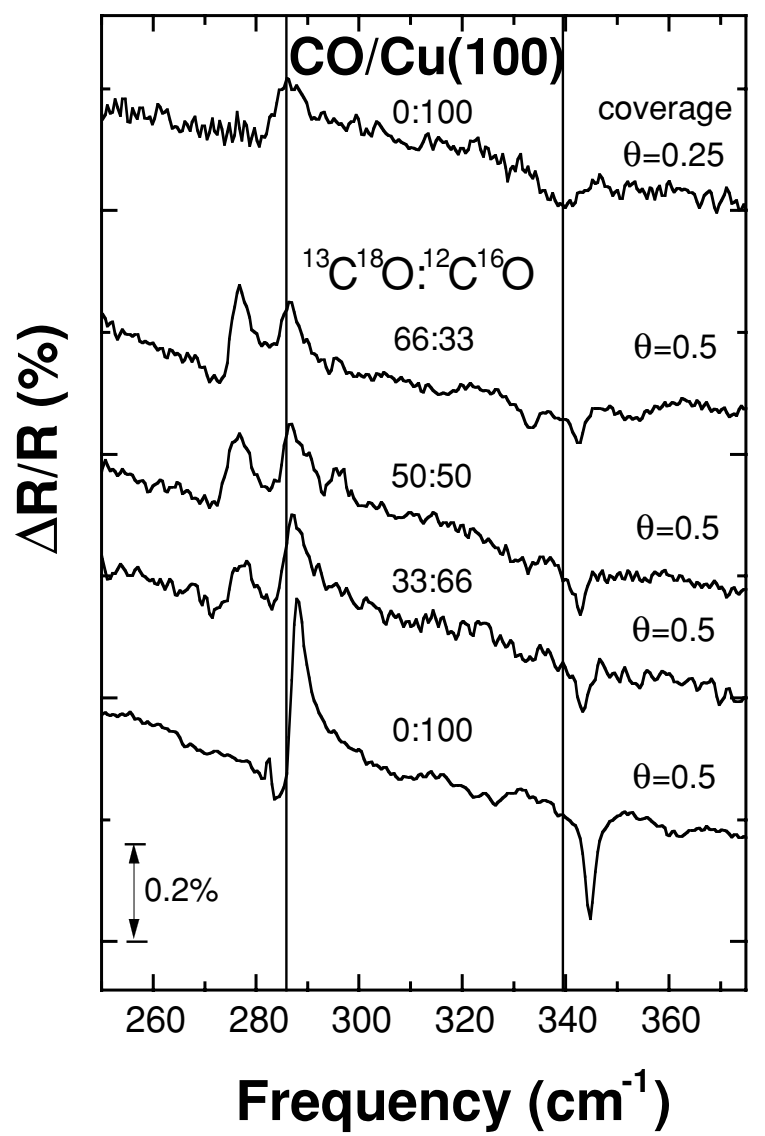

Figure 15. Reflectance changes induced by isotopic mixtures of $\mathrm{CO}$ on a $\mathrm{Cu}\left(\begin{array}{lll}1 & 0 & 0\end{array}\right)$ surface for a total coverage of 0.5 monolayers. A lower coverage spectrum is also shown.

magnitude of the frequency shift between the dilution limit frequency and the homogeneous limit frequency.

Referring to figure 15, the singleton frequency is close to that seen for the top spectrum, the dilution limits are close to that observed for the second to the top spectrum, and the homogeneous limit frequency is that observed for the bottom spectrum. For the analysis we determined both the singleton and the dilution limits by extrapolation of the data to zero coverage since it was not possible to extend the measurements to as low a coverage or dilution limit as for the internal stretch data. The results are shown in figure 17.

In contrast to the intramolecular modes, the low frequency modes were found to be quite sensitive to the bonding environment and to show strikingly different behaviour for the (lll 111$)$ and (100) surfaces [36]. For the $\mathrm{Cu}(100)$ surface the observed chemical shifts for the carbonmetal and hindered rotation modes were $+2.1 \%$ and $+0.7 \%$, respectively. These shifts are in the opposite direction from that of the $\mathrm{C}-\mathrm{O}$ stretch, and indicate that the carbon-metal bond and, to a lesser degree, the hindered rotational mode strengthen with coverage as expected for dative bonding. For the (1 111 ) surface on the other hand, the carbon-metal mode was found to shift by $+0.89 \%$, again indicating bond strengthening but to a lesser degree than for the (100) surface. However, the hindered rotational mode shifted by $-2.2 \%$ with coverage, indicating 
- ISOTOPE A

O ISOTOPE B

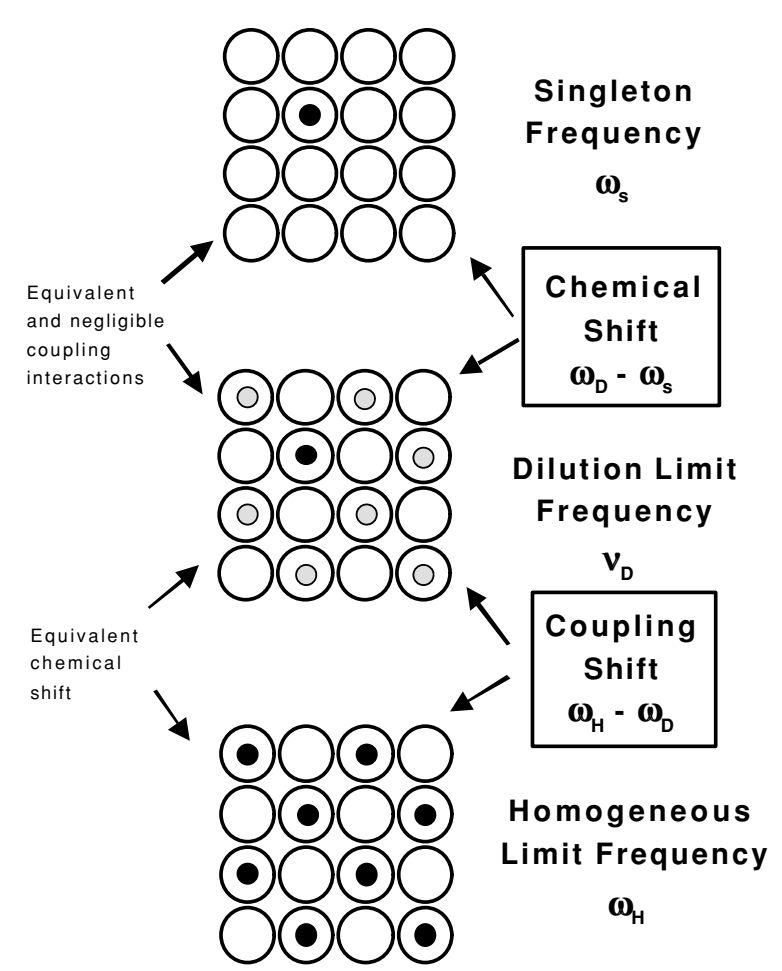

Figure 16. Cartoon illustrating the method of isotopic mixtures for separating chemical from inter-molecular coupling, two effects which are responsible for the coverage dependent frequency shifts of vibrational modes of adsorbates at surfaces.

mode weakening. Thus differences in chemical shifts are apparent between the two surfaces. Both the carbon-metal and the hindered rotation modes are involved in the bonding since both involve motions primarily of the carbon atom adjacent to the surface.

In the analysis of these data the vibrational polarizabilities were determined. This could be done in two ways, from the magnitude of the peaks and from their positions, or more specifically from their shifts. The agreement between the values determined by the two methods is only satisfactory, indicating some inadequacies in the theoretical understanding, which was not evident in the previous investigations using intra-molecular modes.

\section{Conclusions}

We have tried to describe the present situation in which developments in infrared synchrotron radiation have been applied to the study of surface dynamics, which includes vibrational dynamics, but as a sub-set. Thus, new insights into the bonding vibrations and low frequency dynamical behaviour of metals with adsorbates have been obtained. These include the interesting interplay between electrons and vibrational modes, including the observed breakdown of the Born-Oppenheimer approximation. The work has led to an understanding 


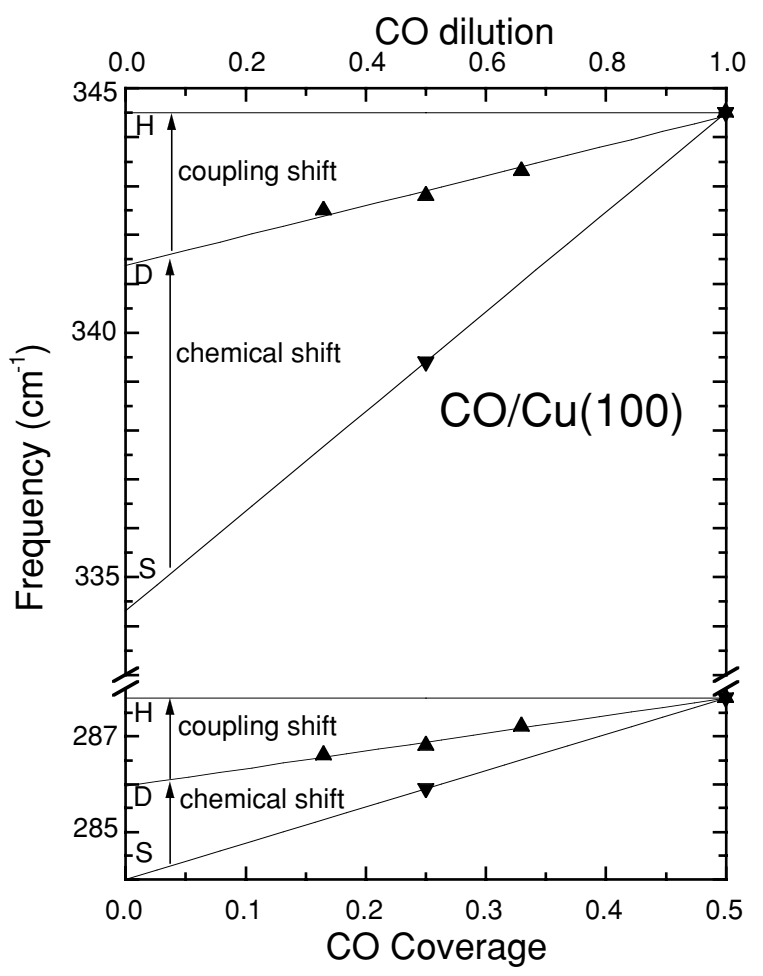

Figure 17. Measured frequency shifts for the bonding and hindered rotational modes (carbonmetal and frustrated rotation) for $\mathrm{CO}$ on $\mathrm{Cu}(100)$, showing the derivation of the coupling and chemical shifts from the data of figure 15 .

of atomic friction and catalysis mechanisms via interaction cross sections and opened up a vast new field for future study.

With the development of even brighter and more stable IR sources this work is expected to continue to provide a deeper understanding of the properties and functions of surfaces, which will always play a critical role in material interactions.

Many new phenomena have been discovered, which have found a strong theoretical basis, but one which also throws up a challenge for further investigation. It is clear that synchrotron radiation has played a strong role in the development of this field, and many of the synchrotron experiments have motivated laboratory-based ones. The field is rich, of much more than academic interest, being highly relevant to many diverse areas of technology, and it will continue.

\section{Acknowledgments}

This work would not have been possible without the dedicated help of the many collaborators who visited Brookhaven between 1984 and 2001. By highlighting some of those, I do not mean to ignore any. Initially involved in the project motivation and early experiments were Yves Chabal and Fritz Hoffmann, while Eric Kneedler assisted in constructing the first beamline. It was a real pleasure to work with Carol Hirschmugl, whose indefatigable, highly motivated and dedicated attitude was responsible for the major evolution of this field from its beginning. 
She had to deal with many of the early problems and was motivated by the excitement of the science and was eventually rewarded, but only after a considerable period. It is also a great pleasure to acknowledge my colleague Paul Dumas with whom I have shared many laboratory hours and discussions and who has been my main partner in developing not only the science discussed here, but also other experiments including microspectroscopy. Without Bo Persson, with whom the interplay between experiment and theory has been as pleasurable as it has been productive, the field would not have progressed as it has. It is also a pleasure to acknowledge those who shared in the development of the ideas, Roger Tobin (and his group, including Kathy Lin), Christine Lamont, Petra Rudolf, Andreas Otto and his group including Matthias Hein who set up the resistivity experiments. I have benefitted enormously by interactions with Larry Carr, and with the NSLS machine group headed by Sam Krinsky. This work would not have been possible without the technical and engineering support of the NSLS and in particular the help of D Carlson, J Gallagher, R Greene, G Nintzel and D Lynch. The NSLS is supported by the United States Department of Energy under contract DE-AC0298-CH10886. Jefferson Lab is supported by the United States Department of Energy under contract DE-AC05-84-ER40150.

\section{References}

[1] Yates J T and Madey T E (ed) 1987 Vibrational Spectroscopy of Molecules on Surfaces (New York: Plenum)

[2] Clark R J H and Heston R E (ed) 1988 Spectroscopy of Surfaces (New York: Wiley)

[3] Hoffmann F M 1983 Surf. Sci. Rep. 3107

[4] Chabal Y J 1988 Surf. Sci. Rep. 8211

[5] Conf. Proc. 'Vibrations at Surfaces'-8 1996 Surf. Sci. 368

[6] Beckerle J D, Casassa M P, Heilweil E J, Cavanagh R R and Stephenson J C 1990 J. Electron Spectrosc. 54/55 17

[7] Chiang S, Tobin R G, Richards P L and Thiel P A 1984 Phys. Rev. Lett. 52648

[8] Duncan W D and Williams G P 1983 Appl. Opt. 222914

[9] Williams G P 2001 Handbook of Vibrational Spectroscopy ed J M Chalmers and P R Griffiths (New York: Wiley) at press

[10] Hirschmugl C J and Williams G P 1995 Rev. Sci. Instrum. 661487

[11] Yu L H, Bozoki E, Galayda J, Krinsky S and Vignola G 1986 Nucl. Instrum. Methods A 246165

[12] Biscardi R, Ramirez G and Williams G P 1995 Rev. Sci. Instrum. 661856

[13] Carr G L, Dumas P, Hirschmugl C J and Williams G P 1988 Nuovo Cimento D 20375

[14] Hirschmugl C J, Williams G P, Chabal Y J and Hoffmann F M 1994 J. Vac. Sci. Technol. A 122229

[15] Hein M, Dumas P, Otto A and Williams G P 2000 Surf. Sci. 465249

[16] Graham A P, Hofmann F, Toennies J P, Williams G P, Hirschmugl C J and Ellis J 1988 J. Chem. Phys. 108 7825

[17] Persson B N J and Volokitin A I 1994 Surf. Sci. 310314

[18] Persson B N J 1991 Phys. Rev. B 443277

[19] Persson B N J, Schumacher D and Otto A 1991 Chem. Phys. Lett. 178204

[20] Lamont C L A, Persson B N J and Williams G P 1995 Chem. Phys. Lett. 243249

[21] Greuter F and Plummer E W 1983 Solid State Commun. 4837

[22] Wissman P 1975 Surface Physics—Springer Tracts in Modern Physics ed G Höhler (Berlin: Springer)

[23] Wahnström G 1989 Chem. Phys. Lett. 163401

[24] Li Y and Wahnström G 1992 Phys. Rev. Lett. 683444

[25] Hellsing B and Persson M 1984 Phys. Scr. 29360

[26] Hoffmann F M, Persson B N J, Walter W, King D A, Hirschmugl C J and Williams G P 1994 Phys. Rev. Lett. 721256

[27] Lin K, Tobin R and Dumas P 1995 J. Vac. Sci. Technol. 131579

[28] Dumas P, Suhren M, Chabal Y J, Hirschmugl C J and Williams G P 1997 Surf. Sci. 371200

[29] Kovar M, Kasza R V, Griffiths K, Norton P R, Williams G P and van Campen D 1998 Surf. Rev. Lett. 5589

[30] Rudolf P, Raval R, Dumas P and Williams G P 2001 Appl. Phys. A submitted

[31] Pilling M, Gardner P, Kauser R, Premble M E and Surman M 1999 Surf. Sci. 433-435 22

[32] Krastner E T, Kuhl D E and Tobin R G 1997 Surf. Sci. 387 L1051 
[33] Kuhl D E, Lin K C, Chung C, Luo J S, Wang H and Tobin R G 1996 Chem. Phys. 2051

[34] Hirschmugl C J private communication

[35] Hoffmann F M, Lin K, Tobin R, Hirschmugl C J, Williams G P and Dumas P 1992 Surf. Sci. Lett. 275 L675

[36] Hirschmugl C J and Williams G P 1995 Phys. Rev. B 5214177

[37] Wang X D, Tysoe W T, Greenler R G and Truszkowska K 1991 Surf. Sci. 257335

[38] Hollins P and Pritchard J 1979 Surf. Sci. 89486

[39] Ryberg R 1982 Surf. Sci. 114627

[40] Persson B N J and Ryberg R 1981 Phys. Rev. B 246954 